\title{
DEPOLARIZATION RATIO OF CLOUDS MEASURED BY MULTIPLE-FIELD OF VIEW MULTIPLE SCATTERING POLARIZATION LIDAR
}

\author{
Hajime Okamoto $^{1 *}$, Kaori Sato ${ }^{1}$, Toshiyuki Makino ${ }^{1}$, Tomoaki Nishizawa ${ }^{2}$, Nobuo Sugimoto \\ Yoshitaka Jin ${ }^{2}$, Atsushi Shimizu ${ }^{2}$ \\ ${ }^{1}$ Research Institute for Applied Mechanics, Kyushu University, Kasuga, Fukuoka 816-8580, Japan, \\ *Email: okamoto@riam.kyushu-u.ac.jp \\ ${ }^{2}$ National Institute for Environmental Studies, Tsukuba, Ibaraki 305-8506, Japan
}

\begin{abstract}
We have developed the Multiple Field of view Multiple Scattering Polarization Lidar (MFMSPL) system for the study of optically thick low-level clouds. It has 8 telescopes; 4 telescopes for parallel channels and another 4 for perpendicular channels. The MFMSPL is the first lidar system that can measure depolarization ratio for optically thick clouds where multiple scattering is dominant. Field of view of each channel was $10 \mathrm{mrad}$ and was mounted with different angles ranging from $0 \mathrm{mrad}$ (vertical) to $30 \mathrm{mrad}$. And footprint size from the total FOV was achieved to be close to that of Cloud-Aerosol Lidar and Infrared Pathfinder Satellite Observation (CALIPSO) lidar at the altitude of $1 \mathrm{~km}$ in order to reproduce similar degree of multiple scattering effects as observed from space. The MFMSPL has started observations since June 2014 and has been continuously operated at National Institute for Environmental Studies (NIES) in Tsukuba, Japan. Observations proved expected performance such that measured depolarization ratio was comparable to the one observed by CALIPSO lidar.
\end{abstract}

\section{INTRODUCTION}

Conventional ground-based lidar has a limited capability to detect low-level clouds due to strong attenuation of lidar signals and maximum optical thickness is about 3 . On the other hand, spaceborne lidar such as CALIPSO lidar can measure signals from deeper part of the clouds. And the depolarization ratio for low-level clouds measured by the CALIPSO lidar often exceeded $30 \%$ while ground-based lidar usually showed smaller than $10 \%$ of depolarization ratio [1]. The difference in depolarization ratio between ground-based conventional lidar and space-borne lidar attributed to the different foot print size between the two. That is, the footprint size of conventional groundbased lidar with FOV of $1 \mathrm{mrad}$ is about $1 \mathrm{~m}$ at the altitude of $1 \mathrm{~km}$ while that of CALIPSO lidar was 90m. Analysis of CALIPSO lidar data showed depolarization ratio of water clouds tended to increase as estimated extinction of the layer increased [2]. The characteristics were reproduced by the results of numerical simulations by Monte Carlo method. And the depolarization ratio of optically thick water clouds becomes comparable to that of randomly oriented ice particles. Due to these characteristics, the discrimination of cloud particle phase was not possible by depolarization information alone in case of space borne lidar observations. Thus it was necessary to take into account the combination of attenuation of backscattered lidar signals and depolarization to discriminate between randomly oriented ice, horizontally oriented ice particles and water particles [2]. It is also worth to note that there is no global product of three-dimensional distribution of water cloud microphysics using CALIPSO data.

There were several attempts to overcome the limitation of detectability of optically thick clouds in the ground-based lidar measurements. Offbeam lidar was introduced to detect lidar signals that were strongly affected by multiple scattering [3,4 and 5]. These have much wider field of view than the conventional lidar and were especially suited to detect multiple scattering signals from water clouds. It is also noted that no ground-based lidar that can detect depolarization ratio of clouds comparable to the observed values by CALIPSO lidar have been built. 
Main aims of the study are to develop a new lidar system than can detect depolarization ratio from optically thick parts of the clouds. Multi-field of view lidar system was introduced with each field of view of $10 \mathrm{mrad}$ and resultant total FOV is $40 \mathrm{mrad}$. The foot print size at the altitude of $1 \mathrm{~km}$ was close to that of CALIPSO so that similar degree of multiple scattering effects was expected in the new lidar system.

\section{Description of the Multiple-Field of view Multiple Scattering Polarization Lidar}

ND:YAG laser was used in the system and only the second harmonic wavelength $(532 \mathrm{~nm})$ of the laser is detected. And the new lidar consists of 8 telescopes to achieve total FOV of $30 \mathrm{mrad}$ and FOV of each telescope is chosen to be $10 \mathrm{mrad}$ to assure the same polarization signatures. Four telescopes for parallel channels are located in a line and angles from the vertical direction are 0,10,20 and $30 \mathrm{mrad}$ for channels $1,3,5$ and 7, respectively. Another four telescopes for perpendicular channels (channels 2, 4, 6 and 8) are also located in a line parallel to the previous line and the angles were chosen to be the same as parallel channels. Each channel consists of polarizer, interference filter, lens and detector where photo multiplier tubes (PMT) are used. The temporal and vertical resolutions of original data were 10 seconds and $6 \mathrm{~m}$, respectively.

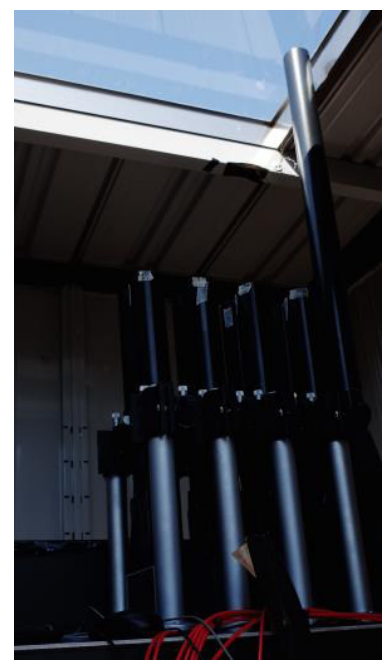

Fig.1. The Multiple Field of view Multiple Scattering Polarization Lidar operated at National Institute for Environmental Studies in Tsukuba since June 2014.
Signal to noise ratio of the MFMSPL system was not sensitive enough to detect signals from upper layers for the calibration target. Therefore calibration of the each channel of MFMSPL was conducted as follows. There were two steps for the calibration; the first one was the absolute calibration of channel 1 (vertical) by the comparison of the collocated National Institute for Environmental Studies (NIES) dual wavelength polarization lidar. Prior to the absolute calibration of the MFMSPL, the NIES lidar was calibrated using signals of upper layers than $6 \mathrm{~km}$. The data for aerosol region observed in June 5, 2014 were chosen for the absolute calibration because there is in generally negligible impact of multiple scattering on the lidar backscattering signals from aerosols at $532 \mathrm{~nm}$. The second calibration procedure is about relative calibration of all channels of the MFMSPL. All 8 telescopes were set to be parallel to vertical direction and polarizers for even channels were not used for the calibration purpose. Then data for aerosol regions were used, too. The calibration was conducted relative to channel 1 .

Cloud mask methods for the off-beam channels (from channel 3 to 8) were different from that for the on-beam channels (channel 1 and 2). Cloud mask scheme for on-beam channels were developed similar to the one for ground-based lidar and also for CALIOP lidar [6,7] where discrimination of clouds, aerosols, molecules and noise were performed. And the cloud mask for off-beam channels were done by discrimination between noise and clouds since signals from offbeam channels were not influenced by aerosols and molecules.

\section{OBSERVATIONAL RESULTS}

The lidar has been continuously operated since June 2014. We showed the preliminary results from observations of low-level clouds on 9 June 2014. Apparent (attenuated) backscattering coefficient coefficient for each parallel channel were shown in Fig. 2. 


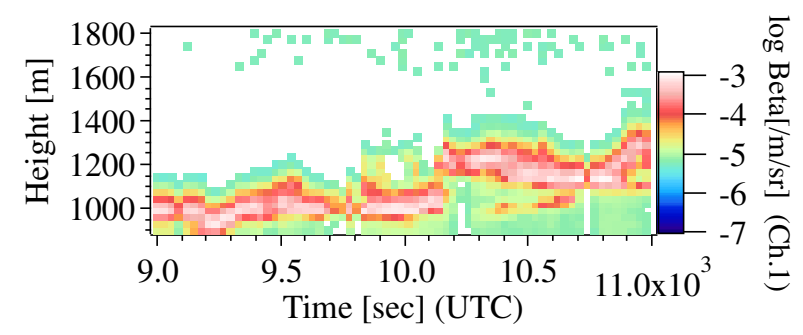

(a)

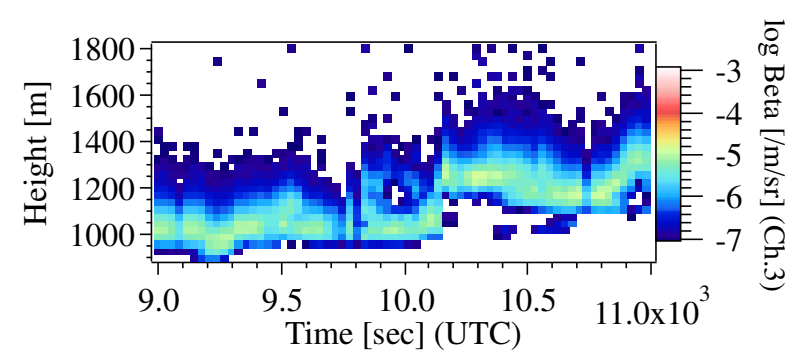

(b)

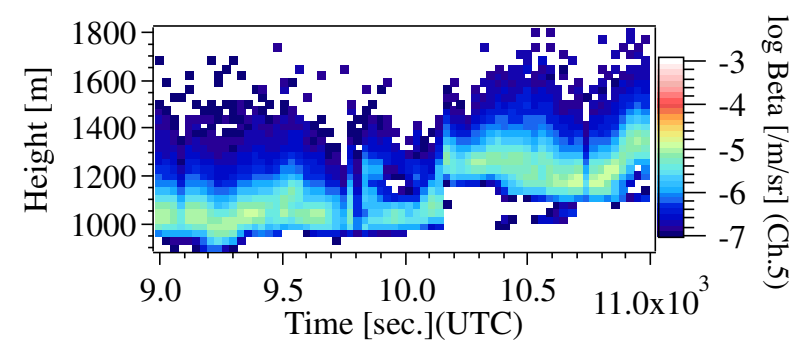

(c)

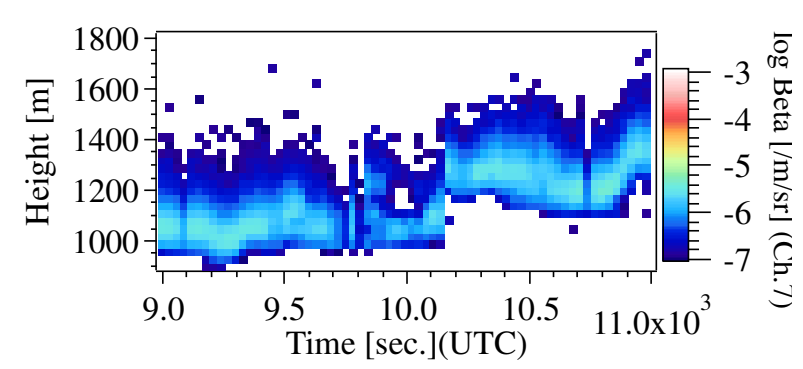

(d)

Fig.2. Time-height plot of apparent backscattering coefficient for parallel channels 1,3,5 and 7. As channel number becomes larger, the direction of the receiver deviated from the direction of laser beam.

Cloud top height $(\mathrm{CTH})$ tended to be higher for outer channels as expected. The CTH for on-beam channel (Ch.1) was lower than $1.2 \mathrm{~km}$ at about 9000 seconds and it increased to $1.4 \mathrm{~km}$ at 10500 seconds. The CTH of the same clouds detected by channel 3 was generally higher than that detected by channel 1 , e.g., $1.4 \mathrm{~km}$ at 9000 seconds and $1.6 \mathrm{~km}$ at 10500 seconds.

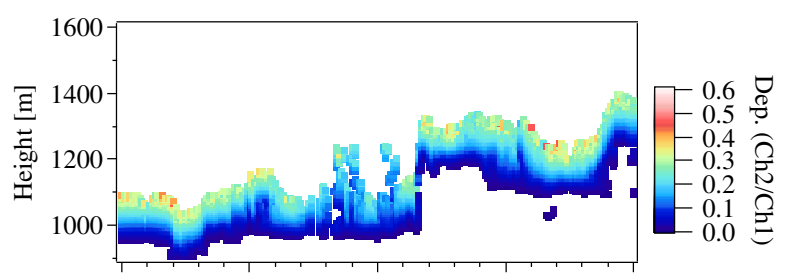

(a)

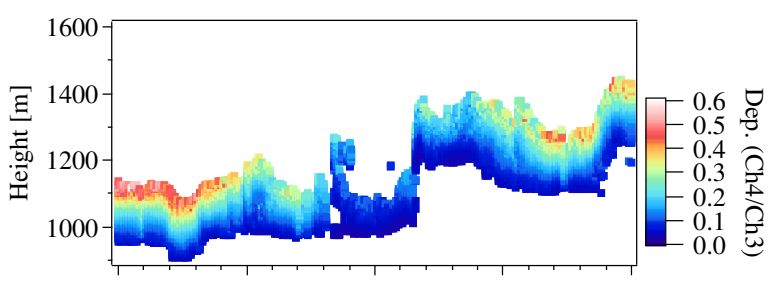

(b)

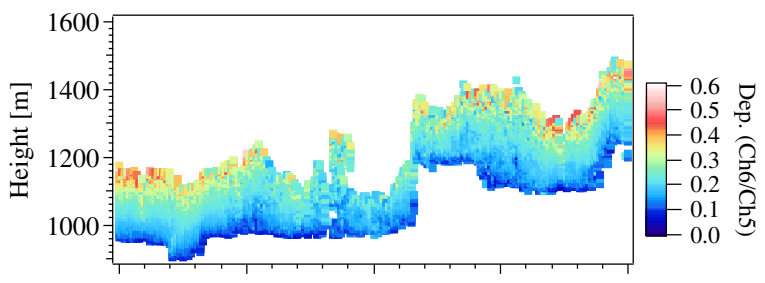

(c)

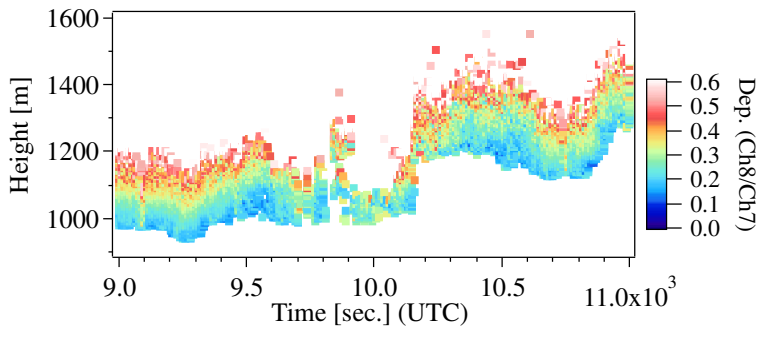

(d)

Fig.3. Same as Fig.3 but for depolarization ratio. Dep. $(\mathrm{Ch} 1 / \mathrm{Ch} 2)$ in the figure denotes depolarization ratio was estimated by using channels 1 and 2 .

The depolarization ratio was also estimated for each set of parallel and perpendicular channels (Fig. 3). The depolarization ratio increased as altitude increased. The value estimated using channel 7 and 8, corresponding to the angle of 30 $\mathrm{mrad}$, often reached $50 \%$. Therefore, it was suggested that the MFMSPL have a potential to 
capture the similar feature found in the CALIPSO lidar observations of optically thick clouds where multiple scattering contribution was significant.

\section{SUMMARY}

We have developed the new type of ground-based lidar, MFMSPL, that can observe depolarization signals from optically thick clouds. It has 8 telescopes for detectors; 4 for parallel and 4 for perpendicular channels. The total FOV was about $30 \mathrm{mrad}$. The lidar has started observations since June 2014. The MFMSPL was designed to achieve similar footprint size of CALIPSO lidar at altitude of $1 \mathrm{~km}$. The MFMSPL successfully detected much higher cloud top compared with that by conventional lidar at the same place. The depolarization ratio from off-beam channel was comparable to the values detected by the CALIPSO lidar. Co-located observations of the MFMSPL and $95 \mathrm{GHz}$ cloud radar have been conducted from February and April in 2015. Analysis of the MFMSPL and the cloud radar would provide a unique opportunity to develop and evaluate the performance of the system. And it is also expected that the system can help to develop the retrieval algorithm for water microphysics using CALIPSO and CloudSat data as previously conducted for the ice microphysics [8]. The JAXA-ESA mission EarthCARE was scheduled to be launched in 2018 and the EarthCARE will carry high spectral resolution lidar at $355 \mathrm{~nm}$ and $94 \mathrm{GHz}$ Doppler cloud radar. Using the MFMSPL data, similar development should be possible for the new mission. The MFMSPL can also provide the fundamental data for the study of interaction between clouds and aerosols.

\section{ACKNOWLEDGEMENT}

This work was supported in part by the JSPS Kakenhi Grant Numbers 25247078 and 15K17762 and also supported by the Collaborated Research Program of Research Institute for Applied Mechanics, Kyushu University.

\section{REFERENCES}

[1] Hu, Y., D. Winker, M. Vaughan, B. Lin, A. Omar, C. Trepte, D. Flittner, P. Yang, S. L.

Nasiri, B. Baum, W. Sun, Z. Liu, Z. Wang, S.
Young, K. Stamnes, J. Huang, R. Kuehn and R. Holz, 2009: CALIPSO/CALIOP Cloud Phase Discrimination Algorithm, J. Atmos. Oceanic Technol., 26, 2293-2309, doi: /10.1175/2009JTECHA1280.1.

[2] Yoshida, R., H. Okamoto, Y. Hagihara and H. Ishimoto, 2010: Global analysis of cloud phase and ice crystal orientation from Cloiud-Aerosol Lidar and Infrared Pathfinder Satellite Observation (CALIPSO) data using attenuated backscattering and depolarization ratio, $J$. Geophys. Atmos., 115, D00H32, doi:1029/2009JD012334.

[3] Polonsky, N. I., S. P. Love and A. B. Davis, 2005: Wide-Angle Imaging Lidar development at the ARM Southern Great Plains site: Intercomparison of cloud property retrievals, $J$. Atmos. Oceanic Technol., 22, 628-648.

[4] Davis, B. A. 2008: Multiple-scattering lidar from both sides of the clouds: Addressing internal strudcture, J. Geophys. Res., 113, D14S10, doi:10.1029/2007JD009666.

[5] Caharan, R. F., M. McGill, and J. Kolansinski, 2005: THOR-Cloud thickness from offbeam lidar returns, J. Atmos. Oceanic Technol., 22, 605-627.

[6] Okamoto, H., T. Nishizawa, T. Takemura, H. Kumagai, H. Kuroiwa, N. Sugimoto, I. Matsui, A. Shimizu, S. Emori, A. Kamei and T. Nakajima, 2007: Vertical cloud structure observed from shipborne radar and lidar: Midlatitude case study during the MR01/K02 cruise of the research vessel Mirai, J. Geophys. Res., 112, D08216, doi:10.1029/2006JD007628.

[7] Hagihara, Y., H. Okamoto and R. Yoshida, 2010: Development of a combined CloudSatCALIPSO cloud mask to show global cloud distribution, J. Geophys. Res., 115, D00H33, doi:10.1029/2009JD012344.

[8] Okamoto, H., K. Sato and Y. Hagihara, 2010: Global analysis of ice microphysics from CloudSat and CALIPSO: intercomparison of specular reflection in lidar signals, J. Geophys. Res., 115, D22209, doi:10.1029/2009JD013383. 